\title{
PROPRIEDADES ELÉTRICAS DOS FILMES DE POLISSACARÍDEOS
}

\section{ELECTRICAL PROPERTIES OF POLYSACCARID FILMS}

\author{
R. A. de SOUSA ${ }^{1}$, R. M. ALMEIDA ${ }^{1}$, C. C. SILVA ${ }^{3}$, e A. A. M. MACÊDO ${ }^{1}$ \\ ${ }^{1}$ Instituto Federal do Maranhão, Laboratório de Pesquisa, Brasil \\ ${ }^{2}$ Universidade Federal do Maranhão, Departamento de Engenharia de Alimentos, Brasil \\ E-mail: rhayson.almeida@gmail.com
}

\author{
article info \\ Article history: \\ Received 2017-09-04 \\ Accepted 2017-11-20 \\ Available online 2017-12-20
}

PALAVRAS-CHAVE: Galactomanana; Quitosana; Blenda; ImpedânciA.

KEYWORDS: Galactomannan; Chitosan; Blend; Impedance.

RESUMO: Galactomanana (Gal) é um polissacarídeo que possui estrutura linear, constituída principalmente de unidades de D-manopiranose por uma ligação $\beta(1-4)$ com moléculas de D-galactopiranose na cadeia principal unidas por uma ligação glicosídica. A estrutura química da quitosana (Q) é formada por unidades de $\beta(1-4)$ 2-amino-2-deoxi-D-glucose repetidamente. A espectroscopia dielétrica é uma caracterização que estuda a resposta de um material através de um estímulo elétrico, normalmente sendo esta uma corrente alternada, e assim, estudar a parte real e imaginária da impedância e de outros termos dependentes da mesma. Este trabalho tem por objetivo produzir filmes poliméricos usando Galactomanana e Quitosana e caracterizá-los por espectroscopia dielétrica. As soluções dos polissacarídeos foram preparadas a uma proporção de 2\%. Em seguida, as soluções de galactomanana $2 \%$ e a solução de quitosana $2 \%$ foram misturadas e solubilizadas para a obtenção dos filmes de Gal, $Q$ e da blenda GalQ. A blenda da GalQ foi caracterizado por espectroscopia dielétrica. Conclui-se que os filmes apresentam características elétricas dos polímeros isolantes.

\begin{abstract}
Galactomannan (Gal) is a polyssacharide which has a linear structure, consisting mainly of units of D-manopyronose linked by $\beta(1-4)$ with D-galactopyranose molecules in the backbone attached by a glycosidic bond. The chemical structure of chitosan (Q) is formed by units of $\beta$ (1-4) 2-amino-2-deoxy-Dglucose repeatedly. Dielectric spectroscopy is a characterization that studies the response of a material through an electrical stimulus, usually being an alternating current, and thus, to study the real and imaginary part of the impedance and other terms dependent on it. This work aims to produce polymer films using Galactomannan and Chitosan and characterize them by dielectric spectroscopy. Then, solutions of $2 \%$ galactomannan and $2 \%$ chitosan solution were mixed and solubilized to obtain Gal, $Q$ and GalQ films. We conclude that the electronic products of the insulating polymers.
\end{abstract}




\section{INTRODUÇÃO}

\subsection{Galactomanana (Gal)}

Encontra-se em paredes de endospermas de sementes da família Leguminosae, tendo função de armazenamento de energia (Gidley; Reid, 2006). Sua estrutura química é constituída por cadeia linear D-manopiranose unidas por ligação $\beta$ (1-4) com ramificações de de D-galactopiranose unidas por ligação glicosídica $\alpha$ (1-4) (Mesquita et al., 2009), conforme Figura 1. Uma das fontes de galactomanana é a Adenanthera pavonina L., árvore da família Fabacea e subfamília Mimosoideae, nativa da Ásia tropical e conhecida como olho-de-dragão, falso pau brasil, Carolina (FONSECA; PEREZ, 2003).

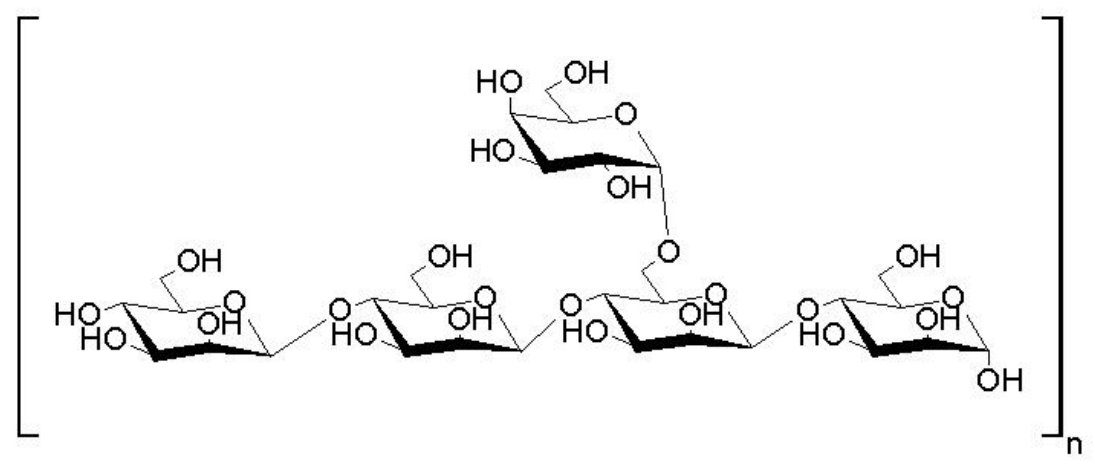

Figura 1 - Estrutura da Galactomanana

\subsection{Quitosana (Q)}

Polissacarídeo amino, encontrado principalmente no exoesqueleto dos crustáceos, é o segundo composto mais abundante na natureza. A quitina é um composto inerte e insolúvel, enquanto quitosana sofre reação e solubilidade em ácidos fracos, caracterizando se assim, como um polieletrólito catiônico. A estrutura química (Figura 2) da quitosana é constituída por unidades de $\beta(1-4)$ 2-amino-2-deoxi-D-glicopiranose repetidamente, unidas por ligações glicosídicas (ASSIS; SILVA, 2003).

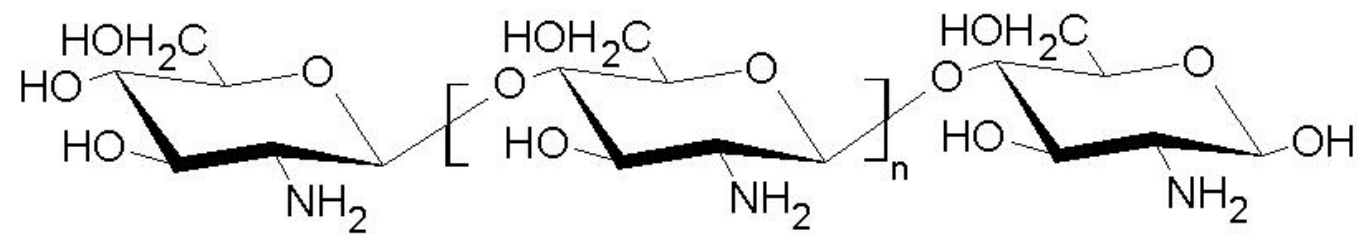

Figura 2 - Estrutura da Quitosana 
O processo de separação da quitina na carapaça dos crustáceos inicia-se com soluções de ácido clorídrico e de hidróxido de sódio que acarretam a desmineralização e desproteinização, sendo que o próximo passo é a descoloração provocada pelo permanganato de potássio e ácido oxálico. A quitina obtida pelo processo é diluída em hidróxido de sódio, sofrendo uma desacetilação e obtendo a quitosana (AZEVEDO et al., 2007).

A quitosana é encontrada na indústria com diversas aplicações, devido suas propriedades biológicas, como: capacidade de ativar a defesa do organismo para combate da doença com o intuito de prevenir infecção e regenerar tecidos, vem sendo usada na medicina veterinária (SENEL, McCLURE, 2016).

\subsection{Espectroscopia de impedância}

Método utilizado para analisar as propriedades elétricas de materiais e sistemas pela indução de um sinal elétrico alternado em diferentes frequências e medindo os sinais de respostas (Zhao et al., 2017). Suas aplicações abrangem diversas áreas, tais como: análises microbiológicas, controle da qualidade de revestimentos, caracterização de sólidos, dentre outras (Grossi; Riccò, 2017). Tomando um estímulo elétrico em determinado material (Equação 1):

$$
v(t)=V \operatorname{sen}(\omega t)
$$

Onde $\mathrm{v}(\mathrm{t})$ é o sinal de entrada [V], $\omega$ é a frequência angular [Hz] e V é a amplitude da voltagem [V]. Obtém-se um sinal de resposta do material (Equação 2):

$$
i(t)=I \operatorname{sen}(\omega t+\varphi)
$$

Sendo i(t) é a corrente de resposta $\mathrm{AC}$ [A], I é amplitude da corrente [A], $\omega$ é a frequência angular $[\mathrm{Hz}]$ e $\varphi$ é o ângulo de defasagem entre o sinal de entrada e o sinal de resposta [rad]. Portanto, a impedância complexa é dada na Equação 3:

$$
Z^{*}(\omega)=v(t) / i(t)=Z^{\prime}+j Z^{\prime \prime}
$$

Onde $Z^{*}(\omega)$ é a impedância complexa do material $[\Omega]$, Z' é a impedância real $[\Omega]$ e Z" é a impedância imaginária $[\Omega]$.

\section{METODOLOGIA}

As sementes de Adenanthera pavonina L. foram submetidas a aquecimento e intumescimento. A separação do endosperma do tegumento ocorreu manualmente, logo após o endosperma foi liofilizado, pulverizado e armazenado à temperatura ambiente (Figura 3). 


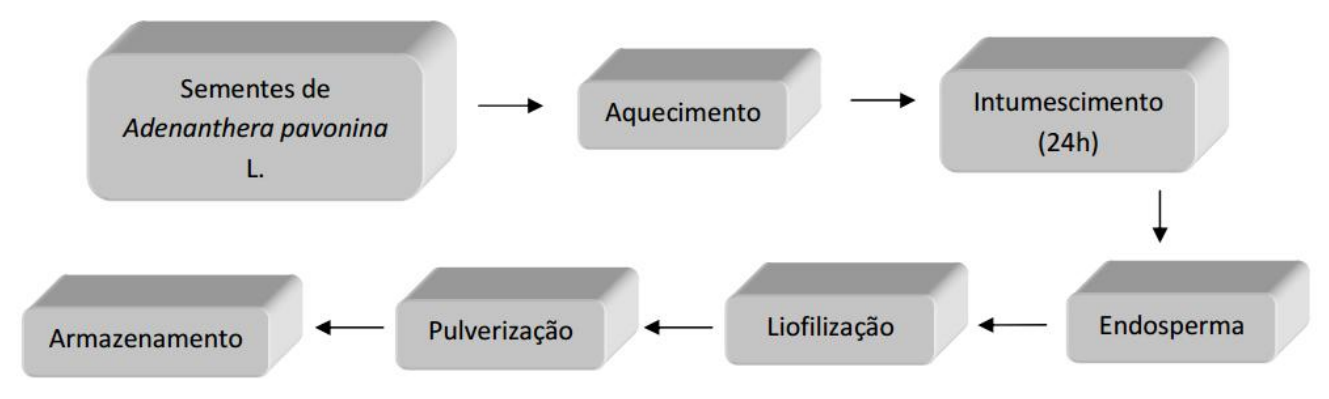

Figura 3 - Metodologia da extração da Galactomanana

Os filmes de Galactomanana, Quitosana e Galactomanana/Quitosana foram formatados por evaporação lenta do solvente, à baixa temperatura.

\section{RESULTADOS E DISCUSSÕES}

$\mathrm{Na}$ figura 4, observa-se apresenta a impedância real de filmes de Galactomanana, Quitosana e Galactomanana/Quitosana.

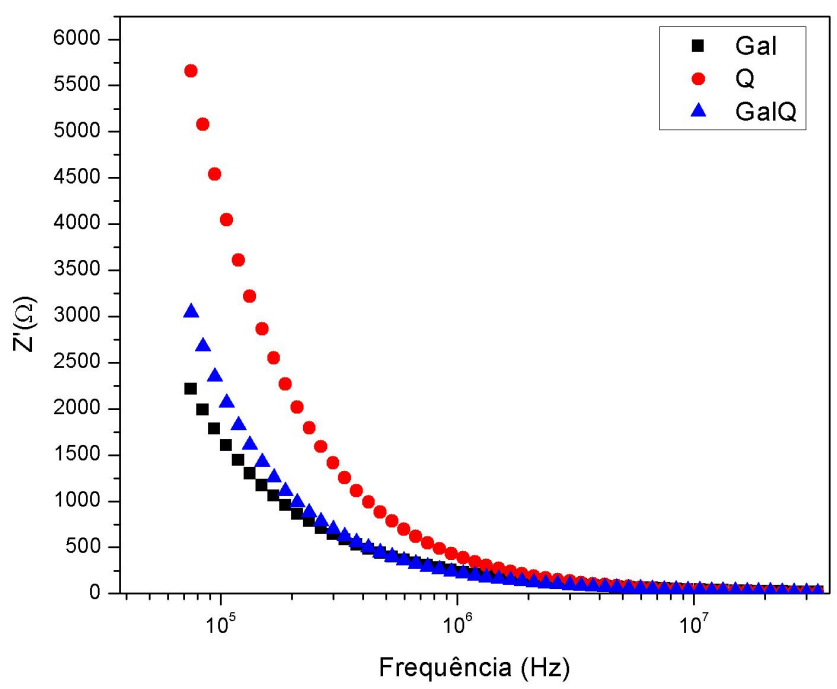

Figura 4 - Impedância real versus frequência dos filmes de Galactomanana, Quitosana e Galactomanana/Quitosana

Observa-se que o filme de quitosana apresenta maior resistência, enquanto que o filme de galactomanana apresenta menor resistência. No filme galactomanana/quitosana, o comportamento dos polissacarídeos foram alterados, apresentando valores intermediários para a parte real da impedância. Estes filmes apresentam uma diminuição exponencial com o 
aumento da frequência. Sugere-se que este efeito é devido ao fenômeno de transporte de cargas do material (Figura 4).

A Figura 5 apresenta a constante dielétrica dos filmes de Galactomanana, Quitosana e Galactomanana/Quitosana.

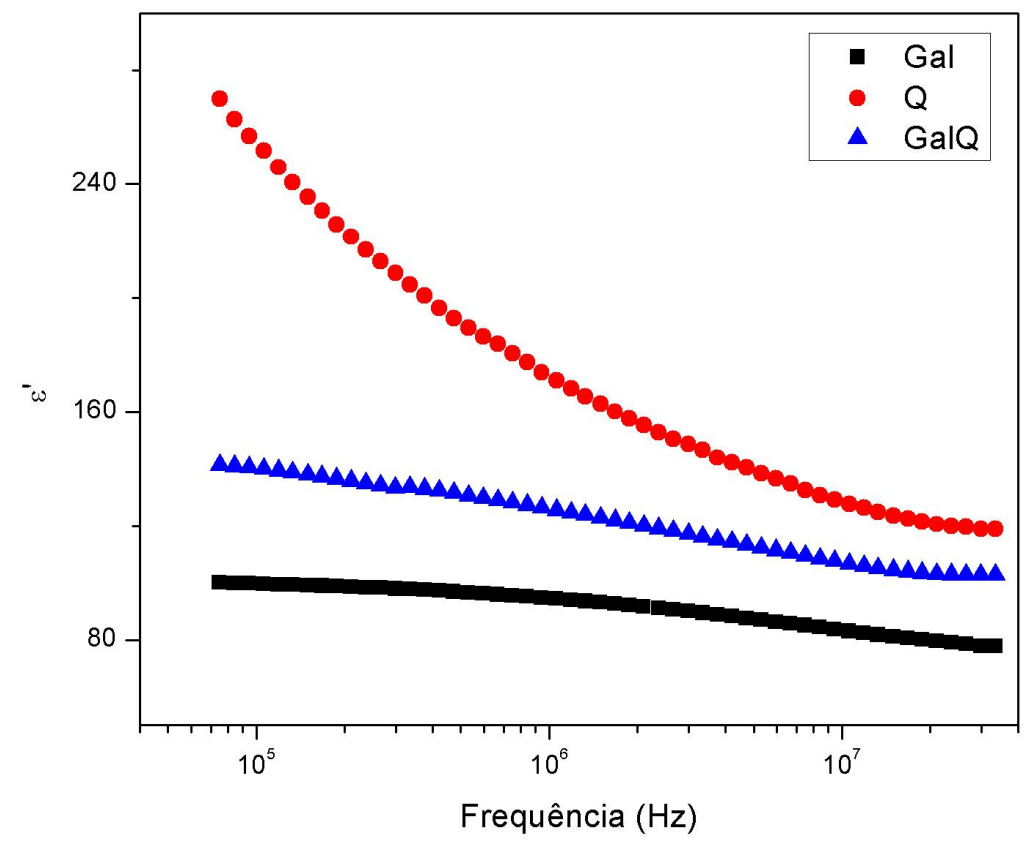

Figura 5 - Constante dielétrica versus frequência dos filmes de Galactomanana, Quitosana e Galactomanana/Quitosana

A constante dielétrica $\left(\varepsilon^{\prime}\right)$ é a habilidade dos materiais polarizarem e armazenarem energia quando submetidas a um campo elétrico externo (Al-oqla, 2015). A Quitosana apresenta maior polarização devido ao seu momento iônico na protonação do grupo amino. Nota-se que a blenda GalQ apresenta valores intermediários para os filmes de Galactomanana e Quitosana. Sugere-se que este efeito esteja relacionado com uma reação química entre elas. Polímeros considerados apolares, como a Galactomanana, devem apresentar uma constante dielétrica entre 2,5 e 3, contudo estes valores divergem dos resultados obtidos. Este aumento na constante dielétrica é resultado da presença de moléculas de água presente na estrutura dos filmes devido às ligações de hidrogênio dos grupos hidroxila (Bibi et al., 2016). Para esta faixa de frequência, a natureza da polarização apresentada nos filmes dos polissacarídeos é da forma dipolar, ou seja, os dipolos acompanham o sentido do campo elétrico (REVIL, 2013).

A Figura 6 mostra a permissividade imaginária versus frequência dos filmes de Galactomanana, Quitosana e Galactomanana/Quitosana. 


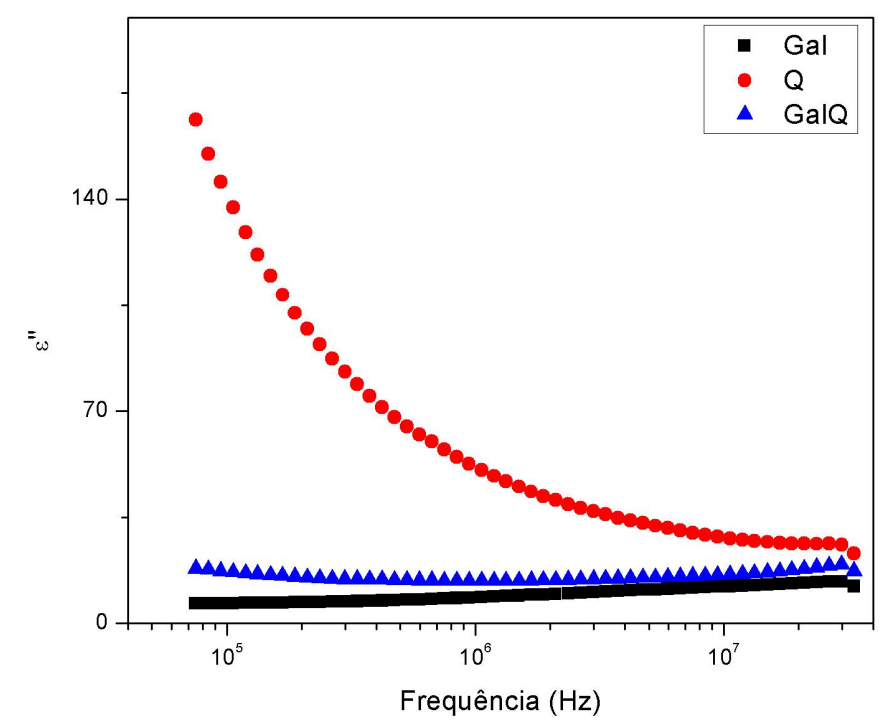

Figura 6 - Permissividade imaginária versus frequência dos filmes de Galactomanana,

Quitosana e Galactomanana/Quitosana

A parte imaginária da permissividade complexa ( $\varepsilon ")$ é usualmente chamada de fator de perda, mostrando que há possíveis efeitos de dissipação de energia (Dang et al., 2012). Percebe-se que a Quitosana apresenta maiores valores para o fator de perda, enquanto que a Galactomanana apresenta menores valores. Observa-se que a forma da perda de energia está associada à condução de cargas nos materiais. Os valores para o fator de perda para filmes. Segundo Bibi e seus colaboradores (2016), os valores esperados para $\varepsilon$ " para uma molécula apolar para esta faixa de frequência é entre $10^{-3}$ a $10^{-4}$. Contudo o aumento observado é do fator de perda é a mesma consequência da presença de moléculas de água na estrutura do material.

\section{CONSIDERAÇÕES FINAIS}

Os filmes de Galactomanana/Quitosana apresentam características intermediárias dos filmes de Galactomanana e Quitosana. As propriedades elétricas destes filmes são próprias de filmes isolantes.

\section{AGRADECIMENTOS}

Os autores são agradecidos a FAPEMA e CNPq. 


\section{REFERÊNCIAS}

AL-OQLA, Faris M. et al. Natural fiber reinforced conductive polymer composites as functional materials: A review. Synthetic Metals, v. 206, p. 42-54, 2015.

BIBI, F. A.; VILLAIN, M.; GUILLAUME, C.; SORLI, B.; GONTARD, N. Review: Origins of the Dielectric Properties of Proteins and Potential Development as Bio-Sensors. Sensors, v. 16, n. 8, p. 1232, 2016.

DANG, Zhi-Min et al. Fundamentals, processes and applications of high-permittivity polymer-matrix composites. Progress in Materials Science, v. 57, n. 4, p. 660-723, 2012.

FERNÁNDEZ-SÁNCHEZ, César; MCNEIL, Calum J.; RAWSON, Keith. Electrochemical impedance spectroscopy studies of polymer degradation: application to biosensor development. TrAC Trends in Analytical Chemistry, v. 24, n. 1, p. 37-48, 2005.

GROSSI, Marco; RICCÒ, Bruno. Electrical impedance spectroscopy (EIS) for biological analysis and food characterization: a review. Journal of Sensors and Sensor Systems, v. 6, n. 2, p. 303, 2017.

PROKSCH, Roger; YABLON, Dalia G. Loss tangent imaging: Theory and simulations of repulsive-mode tapping atomic force microscopy. Applied Physics Letters, v. 100, n. 7, p. 073106, 2012.

RANDVIIR, Edward P.; BANKS, Craig E. Electrochemical impedance spectroscopy: an overview of bioanalytical applications. Analytical Methods, v. 5, n. 5, p. 1098-1115, 2013.

REVIL, A. Effective conductivity and permittivity of unsaturated porous materials in the frequency range $1 \mathrm{mHz}-1 \mathrm{GHz}$. Water Resources Research, v. 49, n. 1, p. 306- 327, 2013.

ZHAO, Xin et al. Electrical Impedance Spectroscopy for Quality Assessment of Meat and Fish: A Review on Basic Principles, Measurement Methods, and Recent Advances. Journal of Food Quality, v. 2017, 2017. 